\title{
Remarks on Ontological Dependence in Set Theory
}

\author{
Thomas Macaulay Ferguson
}

\begin{abstract}
In a recent paper, John Wigglesworth explicates the notion of a set's being grounded in or ontologically depending on its members by the modal statement that in any world (possible or impossible), that a set exists in that world entails that its members exist as well. After suggesting that variable-domain S5 captures an appropriate account of metaphysical necessity, Wigglesworth purports to prove that in any set theory satisfying the axiom Extensionality this condition holds, that is, that sets ontologically depend on their members with respect to extraordinarily weak notions of set. This paper diagnoses a number of problems concerning Wigglesworth's formal argument. For one, we will show that Wigglesworth's argument is invalid as it requires an appeal to hidden, extralogical theses concerning rigid designation and the persistence of sets across possible worlds. Having demonstrated the indispensability of these principles to Wigglesworth's argument, we will then show that even granted the enthymematic premises, the argument only proves the ontological dependence of singletons on their members and does not extend to sets in general. Finally, we will consider strengthenings of Wigglesworth's reasoning and suggest that even the weakest generalization will bear undesirable consequences.
\end{abstract}

\section{Introduction}

That the existence of a set is grounded in the existence of its members is an assertion frequently encountered in discussions of metaphysical grounding ( $c f$. [2] or [8]). Often, this claim is presented as an unambiguous illustration of the phenomenon of grounding. This thesis receives a detailed examination in John Wigglesworth's recent paper [10, in which Wigglesworth sets out to provide a demonstration that a set $S$ ontologically depends on its members. Wigglesworth's characterization of ontological dependence is an asymmetric relation, according to which the existence of a set entails the existence of its members while the converse does not hold. We will not argue against the latter clause, and concern ourselves with the first direction, which is recapitulated in the language of modal logic as:

Ground It is necessary that if a set $S$ exists, then every member of $S$ exists. 
The majority of Wigglesworth's paper is devoted to philosophical arguments but the final pages of the paper are devoted to a formal proof that in every variable-domain S5 model whose elements satisfy very weak conditions about sets, Ground holds with respect to singletons. Moreover, Wigglesworth also speculates that "there is reason to think" [10, 174] that the line of argument provided extends to establish that Ground holds of all sets.

The aim of this paper is to make explicit a number of problems inherent to Wigglesworth's argument. Before identifying the problems, we will first review the argument itself.

\section{Examining Wigglesworth's Argument}

Over the course of [10, Wigglesworth argues that variable-domain first-order S5 corresponds with metaphysical necessity and presumes S5 to serve as the appropriate setting in which to seat talk of necessary properties of sets. We will assume that this is correct in the sequel. The background logic is then defined model-theoretically:

Definition 1. A variable-domain, first-order S5 model is a tuple $\langle D, W, R, v\rangle$ where:

- $D$ is a nonempty set of individuals

- $W$ is a nonempty set of worlds

- $R=W \times W$

- $v$ is a function that

- assigns to each constant term $\underline{t}$ a denotation $t^{\delta} \in D$

- assigns to each $w \in W$ a domain $\mathcal{E}_{w} \subseteq D$

- assigns to each $n$-ary relation symbol $P$ an extension $P_{w} \subseteq D^{n}$

With slight notational modifications, Wigglesworth follows the presentation of [7] with respect to the recursive definition of the forcing relation between worlds and formulae. These conditions are familiar, but for details the reader is referred to 7 .

Wigglesworth understandably wishes to argue that Ground holds with respect to even very weak understandings of set. In particular, Wigglesworth intends for his result to hold for any objects satisfying the axiom of Extensionality, i.e.:

Extensionality $\forall x \forall y(\forall z(z \varepsilon x \leftrightarrow z \varepsilon y) \rightarrow x=y)$

Before recapitulating the argument of [10, a brief note on notation: It is common when writing about formal accounts of set theory to use the same symbols 
in both the formal language and metalanguage. This frequently leads to ambiguity. Rather than use the symbols " $\in$ " and " $\varnothing$ " in both the object and metalanguage, we will employ the symbols " $\varepsilon$ " and " $\underline{\Lambda}$ " in our formal language to denote a binary relation and a constant whose intended interpretations are that of set membership and the empty set, respectively.

\subsection{The Formal Argument}

The structure of Wigglesworth's argument is that of a reductio. A number of prima facie plausible theses are paired with a formal sentence representing the denial of Ground for singletons, and a contradiction is purportedly derived. From this, the reader of [10] is intended to infer that the restriction of Ground to the case of singletons must hold. The initial set-up for the argument as Wigglesworth presents it is that with respect to some model $\mathfrak{M}$ and possible world $w$, the following hold:

$$
\begin{aligned}
& \mathbf{1} w \Vdash \mathcal{E}(\underline{a}) \\
& \mathbf{2} w \Vdash \underline{s}=\imath x . \forall y(y \varepsilon x \leftrightarrow(\mathcal{E}(y) \wedge y=\underline{a})) \\
& \mathbf{3} \text { For all } w^{\prime}, w^{\prime} \Vdash \forall x \forall y(\forall z(z \varepsilon x \leftrightarrow z \varepsilon y) \rightarrow x=y) \\
& \mathbf{4} w \nVdash \square(\neg \mathcal{E}(\underline{a}) \rightarrow \neg \exists x \forall y(y \varepsilon x \leftrightarrow(\mathcal{E}(y) \wedge y=\underline{a})))
\end{aligned}
$$

The natural language equivalents of these assumptions can be described in the following terms:

$\mathbf{1}^{\prime}$ The set $a$ exists at $w$.

$\mathbf{2}^{\prime}$ The set $s$ exists at $w$ and is the unique singleton of $s$ at $w$.

$\mathbf{3}^{\prime}$ Every $w^{\prime}$ is a model of a set theory satisfying Extensionality.

$4^{\prime}$ It is possible that the singleton containing $a$ exists while $a$ does not.

From these assumptions, Wigglesworth aims to prove a contradiction, entailing that no model simultaneously satisfies $\mathbf{1}-\mathbf{4}$.

With inessential changes to the order in which the contradictory consequences are derived, we may easily follow the derivation of the putative contra-

diction. From 4, we are licensed to infer the existence of an accessible world $w^{\prime}$ at which both:

$$
\begin{aligned}
& \mathbf{5} w^{\prime} \Vdash \neg \mathcal{E}(\underline{a}) \\
& \mathbf{6} w^{\prime} \Vdash \exists x \forall y(y \varepsilon x \leftrightarrow(\mathcal{E}(y) \wedge y=\underline{a}))
\end{aligned}
$$

hold. By instantiating 6 on a new constant $\underline{s}^{\prime}$, Wigglesworth infers that:

$$
7 w^{\prime} \Vdash \forall y\left(y \varepsilon \underline{s}^{\prime} \leftrightarrow(\mathcal{E}(y) \wedge y=\underline{a})\right)
$$


Furthermore, as $\mathbf{5}$ has been assumed to hold, there is no possible element of the domain of $w^{\prime}$ that bears the relation $\varepsilon$ to $s^{\prime}$, i.e., we infer that:

$$
8 w^{\prime} \Vdash \neg \exists x\left(x \varepsilon \underline{s}^{\prime}\right)
$$

This is to say that at $w^{\prime}, s^{\prime}$ plays the role of the empty set. Now, Wigglesworth introduces a constant $\underline{\Lambda}$ that by definition denotes the empty set. As we have observed in $\mathbf{8}, \neg \exists x\left(x \varepsilon \underline{s}^{\prime}\right)$ holds at $w^{\prime}$ and by definition $\neg \exists x(x \varepsilon \underline{\Lambda})$ holds at $w^{\prime}$. It follows that $\forall z\left(z \varepsilon \underline{s}^{\prime} \leftrightarrow z \varepsilon \underline{\Lambda}\right)$ is true at $w^{\prime}$ and on this basis, Wigglesworth invokes 3 - the validity of Extensionality - to infer the following:

$$
9 w^{\prime} \Vdash \underline{\Lambda}=\underline{s}^{\prime}
$$

Because $\underline{s}^{\prime}$ and $\underline{\Lambda}$ are constants, these terms are rigid designators, whence we are licensed to infer that:

$$
10 w^{\prime} \Vdash \square\left(\underline{\Lambda}=\underline{s}^{\prime}\right)
$$

From $\mathbf{1 0}$ and the universality of the accessibility relation, we infer that:

$$
11 w \Vdash \underline{\Lambda}=\underline{s}^{\prime}
$$

From 11, Wigglesworth now aims to infer the truth of the following formula at $w$ :

$$
\forall y\left(y \varepsilon \underline{s}^{\prime} \leftrightarrow(\mathcal{E}(y) \wedge y=\underline{a})\right)
$$

It is at this juncture that Wigglesworth begins appealing to unorthodox theses concerning the behavior of rigid designators introduced or baptized by means of a definition. The first instance is when Wigglesworth asserts that because $\underline{s}^{\prime}$ was baptized by means of the description $\imath x . \forall y(y \varepsilon x \leftrightarrow(\mathcal{E}(y) \wedge y=\underline{a}))$, its referent must satisfy the open formula $\forall y(y \varepsilon x \leftrightarrow(\mathcal{E}(y) \wedge y=\underline{a}))$ in any possible world. Such an assertion might be thought of as a persistence criterion, as it describes necessary conditions for a set - in this case, a singleton - to persist across possible worlds.

For the purpose of reviewing Wigglesworth's argument, it suffices to note that the structure of the justification is "because the property $\varphi$ is definitional of $t$, this property should hold of $t$ in every possible world." The matter of the types of properties and objects with respect to which this reasoning is valid will be set aside for the moment. For now, the use of this persistence criterion in the argument is restricted to the case of singletons. A very conservative explication of the persistence criterion justifying this inference can be phrased:

Res- $\mathbf{W}_{\mathbf{1}}$ If a term $\underline{t}$ is introduced by the description $\underline{t}=\imath x . \forall y(y \varepsilon x \leftrightarrow(\mathcal{E}(y) \wedge$ $y=\underline{a}))$ then the formula $\forall y(y \varepsilon \underline{t} \leftrightarrow(\mathcal{E}(y) \wedge y=\underline{a}))$ is true in every possible world at which $\mathcal{E}(\underline{t})$ holds.

Australasian Journal of Logic (13:3) 2016, Article no. 1 
In simpler language, it is necessary that the referent of the denoting phrase "the singleton of $a$ " includes $a$ as a member 1

Returning to the argument, the importance of Res- $\mathbf{W}_{\mathbf{1}}$ is found in its licensing the inference from $\mathbf{1 1}$ to:

$$
12 w \Vdash \forall y\left(y \varepsilon \underline{s}^{\prime} \leftrightarrow(\mathcal{E}(y) \wedge y=\underline{a})\right)
$$

But in virtue of assumption $\mathbf{1}, \mathcal{E}(\underline{a})$ holds at $w$, and it is an easy consequence of $\mathbf{1 2}$ to infer that:

$$
13 w \Vdash \exists x\left(x \varepsilon \underline{s}^{\prime}\right)
$$

To derive the contradiction he seeks, Wigglesworth again appeals to a persistence criterion akin to Res- $\mathbf{W}_{\mathbf{1}}$, this time applying it to the constant $\underline{\Lambda}$ instead of $\underline{s}^{\prime}$. We will describe this principle as follows:

Res- $\mathbf{W}_{\mathbf{2}}$ If a term $\underline{t}$ is introduced by the description $\underline{t}=\imath x . \neg \exists y(y \varepsilon x)$ then the formula $\neg \exists y(y \varepsilon \underline{t})$ is true in every possible world at which $\mathcal{E}(\underline{t})$ holds.

In other words, the formula $\neg \exists y(y \varepsilon x)$ is "definitional" of $\underline{\Lambda}$ as well, as the term $\underline{\Lambda}$ had been introduced as the unique element containing no members. Res- $\mathbf{W}_{\mathbf{2}}$ entails the referent of $\underline{\Lambda}$ (i.e., $\Lambda$ ) will satisfy this formula at any world. Hence, $\Lambda$ will have no members at any world:

$$
14 w \Vdash \neg \exists x(x \varepsilon \underline{\Lambda})
$$

By $\mathbf{1 1}$, the identity $\underline{\Lambda}=\underline{s}^{\prime}$ holds at $w$, whence we cite the properties of identity and substitute $\underline{s}^{\prime}$ for $\underline{\Lambda}$, concluding:

$$
15 w \Vdash \neg \exists x\left(x \in \underline{s}^{\prime}\right)
$$

And 13 and 15 are inconsistent with one another.

It follows, according to Wigglesworth, that the conjunction of assumptions 1-4 is inconsistent and a restricted instance of Ground holds. Wigglesworth infers that every singleton $\{a\}$ ontologically depends on the existence of $a$, and this forms the basis for an informal argument that Ground is true in full generality.

We will now proceed to identify several problems concerning the foregoing argument. First, we will describe a variable-domain S5 model satisfying Wigglesworth's conditions 1-4. The mutual consistency of these theses demonstrates that Extensionality alone does not establish Ground in the case of singletons and shows the indispensability of the hidden premises Res- $\mathbf{W}_{\mathbf{1}}$ and

\footnotetext{
${ }^{1}$ Res- $\mathbf{W}_{1}$ is represented here as a logical or linguistic thesis - rather than a metaphysical thesis - and it is worth considering the justification for this interpretation. If $\mathbf{R e s}-\mathbf{W}_{\mathbf{1}}$ is interpreted as a metaphysical principle - that it is necessary that the set $s$ (i.e., $\{a\})$ contains $a$ as its sole element - then the assumption is just another statement of Ground for singletons. To interpret the assumption in this way would thus assume that Wigglesworth is begging the question, for which reason we default to the linguistic interpretation.
} 
Res- $\mathbf{W}_{\mathbf{2}}$ in Wigglesworth's argument. We will then show that even supposing that Res- $\mathbf{W}_{\mathbf{1}}$ and Res- $\mathbf{W}_{\mathbf{2}}$ hold, Extensionality in conjunction with these two theses remains insufficient to extend the truth of Ground to all sets. Finally, we will consider attempts to strengthen Res- $\mathbf{W}_{\mathbf{1}}$ and $\mathbf{R e s}-\mathbf{W}_{\mathbf{2}}$ to more general principles and we will suggest that even the weakest possible generalization carries consequences orthogonal to Wigglesworth's aims.

\subsection{The Indispensability of Res- $\mathrm{W}_{1}$ and Res- $\mathrm{W}_{2}$}

To show that Wigglesworth must appeal to the principles Res- $\mathbf{W}_{\mathbf{1}}$ and Res$\mathbf{W}_{\mathbf{2}}$, we consider a formal model that meets Wigglesworth's standards yet witnesses an occasion of a singleton existing without its member. This will show that Res- $\mathbf{W}_{\mathbf{1}}$ and Res- $\mathbf{W}_{\mathbf{2}}$ are indispensable to Wigglesworth's proof that Ground holds of singletons.

The model we will employ is very simple, with only two elements in its domain:

Definition 2. The model $\mathfrak{M}_{1}$ is $\langle W, D, R, v\rangle$ where:

- $W$ consists of two worlds $\left\{w, w^{\prime}\right\}$

- $D$ consists of two elements $\{a, s\}$ (denoted by $\underline{a}$ and $\underline{s}$, respectively)

- $R$ is $W \times W$

- $v$ provides the interpretation so that:

$-\mathcal{E}_{w}=D$ and $\mathcal{E}_{w^{\prime}}=\{s\}$

$-\varepsilon_{w}=\{\langle a, s\rangle\}$ and $\varepsilon_{w^{\prime}}=\varnothing$

We will show that insofar as elements $a$ and $s$ satisfy Extensionality, they qualify as sets in the very modest sense Wigglesworth requires and that $\mathfrak{M}$ witnesses a case in which a singleton $(s)$ does not ontologically depend on its member $(a)$. The upshot of this will be that Extensionality alone is not sufficient to ensure the ontological dependence of sets upon their members.

We have noted that Wigglesworth assumes of sets only that they satisfy Extensionality. In any counterexample, this bar must be met by all elements of the domain. Let us first ensure that we are dealing with at least this meager notion of set at all possible worlds:

Observation 1. $\varepsilon$ satisfies Extensionality in both $w$ and $w^{\prime}$.

Proof. In $w$, the universal quantifiers range over $\{a, s\}$. Because $\underline{a}=\underline{a}$ and $\underline{s}=\underline{s}$ are true at $w$, the evaluation of $\forall x, y(\forall z(z \varepsilon x \leftrightarrow z \varepsilon y) \rightarrow x=y)$ thus stands or falls with the truth or falsity of the conjunction of sentences $\forall z(z \varepsilon$ $\underline{a} \leftrightarrow z \varepsilon \underline{s}) \rightarrow \underline{a}=\underline{s}$ and $\forall z(z \varepsilon \underline{s} \leftrightarrow z \varepsilon \underline{a}) \rightarrow \underline{s}=\underline{a}$. But $\forall z(z \varepsilon \underline{s} \leftrightarrow z \varepsilon \underline{a})$ (and $\forall z(z \varepsilon \underline{a} \leftrightarrow z \varepsilon \underline{s})$ ) are false because $\underline{a} \varepsilon \underline{s}$ is true but $\underline{a} \varepsilon \underline{a}$ is not. Hence, Extensionality is true at $w$. 
In $w^{\prime}$, the universal quantifiers range over $\{s\}$. So the evaluation of Extensionality reduces to the evaluation of the sentence $(\underline{s} \varepsilon \underline{s} \leftrightarrow \underline{s} \varepsilon \underline{s}) \rightarrow \underline{s}=\underline{s}$. As $w^{\prime} \Vdash \underline{s}=\underline{s}$, this sentence trivially holds, whence Extensionality holds at $w^{\prime}$.

So at both $w$ and $w^{\prime}$, elements $a$ and $s$ are sets in the meager sense that Wigglesworth requires.

We may proceed to establish some further facts concerning the properties of $\mathfrak{M}_{1}$ and the existence of particular elements of the domain.

Observation 2. At $w, \mathcal{E}(\underline{a})$ and $\mathcal{E}(\underline{s})$ are true.

Proof. By construction of the model, $a$ and $s$ are members of the extension of $\mathcal{E}$ at $w$.

Observation 3. At $w^{\prime}, \neg \mathcal{E}(\underline{a})$ and $\mathcal{E}(\underline{s})$ are true.

Proof. The interpretation of $\mathcal{E}$ at $w^{\prime}$ is the set $\{s\}$, i.e., a does not satisfy the existence predicate $\mathcal{E}$ at $w^{\prime}$ but $s$ does.

Observation 4. At $w^{\prime}, \exists y \forall x(x \varepsilon y \leftrightarrow(\mathcal{E}(x) \wedge x=\underline{a}))$ is true.

Proof. The quantifiers at $w^{\prime}$ range over the set $\{s\}$, so the evaluation of this formula reduces to the evaluation of the sentence $\underline{s} \varepsilon \underline{s} \leftrightarrow(\mathcal{E}(\underline{s}) \wedge \underline{s}=\underline{a})$. Both $\underline{s} \varepsilon \underline{s}$ and $\mathcal{E}(\underline{s}) \wedge \underline{s}=\underline{a}$ are false at $w^{\prime}$, so the sentence asserting their material equivalence is true.

From the foregoing observations, we may also observe that the singleton case of Ground fails:

Observation 5. At $w, \neg \square[\neg \mathcal{E}(\underline{a}) \rightarrow \neg \exists y \forall x(x \varepsilon y \leftrightarrow(\mathcal{E}(x) \wedge x=\underline{a}))]$ is true.

Proof. By Observations 3 and $4 . \neg \mathcal{E}(\underline{a}) \wedge \exists y \forall x(x \in y \leftrightarrow(\mathcal{E}(x) \wedge x=\underline{a}))$ is true at $w^{\prime}$, whence $\neg(\neg \mathcal{E}(\underline{a}) \rightarrow \neg \exists y \forall x(x \varepsilon y \leftrightarrow(\mathcal{E}(x) \wedge x=\underline{a})))$ is true at $w^{\prime}$. As $w R w^{\prime}$, it follows that $\neg \square(\neg \mathcal{E}(\underline{a}) \rightarrow \neg \exists y \forall x(x \in y \leftrightarrow(\mathcal{E}(x) \wedge x=\underline{a})))$ holds at $w$.

At this point, the argument of [10] would have it that $s$ is the unique element satisfying the formula $\neg \exists y(y \varepsilon x)$ at $w^{\prime}$ and infer from this that $s$ must satisfy this formula at $w$ as well. This would then contradict the fact that $s$ has a member at $w$, and the suitability of $\mathfrak{M}_{1}$ as a model would be rejected. But the way in which we have defined the model makes clear that no such move is available.

Within this model, at $w^{\prime}, s$ has the property associated with the definite description "the empty set" and at $w, s$ does not have this property. Although this may at first blush appear to be counterintuitive, there is nothing inconsistent about this scenario.2 "The empty set" is a definite description, and (barring

\footnotetext{
${ }^{2}$ But perhaps this is not so counterintuitive. From the perspective of someone living at $w^{\prime}$, the model reflects an intuition that the empty set could have contained a member. It is not clear that this is any more problematic than an assertion that an empty shoebox could have contained a pair of shoes.
}

Australasian Journal of Logic (13:3) 2016, Article no. 1 
Res- $\mathbf{W}_{\mathbf{2}}$ ) there is no logical requirement that $s$ answers to the same definite description at all possible worlds, any more than there is a requirement that Benjamin Franklin invents bifocals at every possible world.

At this point, we have provided a consistent model witnessing that the formal sentences 1-4 are mutually satisfiable. But some ambiguity remains with respect to the reading of Ground. It will behoove us to clear away this ambiguity before proceeding.

There are two ways that one could construe the sentence "if the singleton of $a$ exists in world $w^{\prime}$ then its member exists in world $w^{\prime}$." In the first case - the de re reading - the description "the singleton of $a$ " is evaluated at the actual world. In the second - the de dicto reading - the description "the singleton of $a$ " is given a local evaluation at each $w^{\prime}$. More precisely, the de re reading can be adequately phrased as:

- The individual satisfying the description "the singleton of $a$ " is such that if it exists at a world $w^{\prime}$, then $a$ exists there as well.

and the de dicto reading can be understood as:

- If at some world $w^{\prime}$ there exists an individual satisfying the description "the singleton of $a$," then $a$ exists there as well.

One might object to the admissibility of the countermodel $\mathfrak{M}_{1}$ by suggesting that the scope of the necessity operator in Ground has been mischaracterized. Anticipating such an objection, we may confirm that both readings fail in the countermodel.

Letting $w$ stand in place of the actual world, on the de re reading, $s$ is the thing that actually satisfies the description $\imath x . \forall y(y \varepsilon x \leftrightarrow(\mathcal{E}(x) \wedge x=\underline{a}))$. Then we observe that the de re account fails:

Observation 6. $w^{\prime} \Vdash \mathcal{E}(\underline{s})$ and $w^{\prime} \nVdash \mathcal{E}(\underline{a})$, i.e., $s$ exists at $w^{\prime}$ while a does not.

Proof. Immediate from construction of the model.

But the de dicto account, which requires that the existence of any referent to the description $\imath x . \forall y(y \varepsilon x \leftrightarrow(\mathcal{E}(y) \wedge y=\underline{a}))$ at $w^{\prime}$ entails the existence of $a$, fares as poorly.

Observation 7. $\left.w^{\prime} \Vdash \mathcal{E}(\urcorner x . \forall y(y \varepsilon x \leftrightarrow(\mathcal{E}(y) \wedge y=\underline{a}))\right)$ and $w^{\prime} \nVdash \mathcal{E}(\underline{a})$, i.e., $\imath x . \forall y(y \in x \leftrightarrow(\mathcal{E}(y) \wedge y=\underline{a}))$ exists at $w^{\prime}$ while a does not.

Proof. At $w^{\prime}$, no element bears $\varepsilon$ to $s$ nor does any element satisfy the formula $\mathcal{E}(x) \wedge x=\underline{a}$. Hence, $\forall x[x \varepsilon \underline{s} \leftrightarrow(\mathcal{E}(x) \wedge x=\underline{a})]$ is true at $w^{\prime}$. As $s$ is the sole element of $\overline{\mathcal{E}}_{w^{\prime}}$ and $s$ satisfies the open formula $\forall y(y \varepsilon x \leftrightarrow(\mathcal{E}(y) \wedge y=\underline{a})$, this entails that $w^{\prime} \Vdash \mathcal{E}(\neg x . \forall y(y \varepsilon x \leftrightarrow(\mathcal{E}(y) \wedge y=\underline{a})))$. As before, we also know that $w^{\prime} \nVdash \mathcal{E}(\underline{a})$.

From these observations, it is clear that appeals to scope provide no aid to Wigglesworth's argument.

Australasian Journal of Logic (13:3) 2016, Article no. 1 


\section{Examining the Persistence Criteria}

The foregoing has been intended to isolate flaws in Wigglesworth's methodology. We have shown that the enthymematic principles Res- $\mathbf{W}_{\mathbf{1}}$ and $\mathbf{R e s}-\mathbf{W}_{\mathbf{2}}$ are in fact necessary components of Wigglesworth's argument, i.e., that absent these extralogical principles, the set of premises Wigglesworth uses for reductio is consistent. In this section, we will prove that the principles $\mathbf{R e s}-\mathbf{W}_{\mathbf{1}}$

and Res- $\mathbf{W}_{\mathbf{2}}$ are insufficient to lift Ground to the case of all sets. Extending Wigglesworth's argument to show that Ground holds in full generality thus requires much stronger assumptions.

\subsection{The Insufficiency of Res- $\mathrm{W}_{1}$ and Res- $\mathrm{W}_{2}$}

As before, we will rely on a variable-domain S5 model in order to prove the consistency of a set of assumptions. In this case, the model to be constructed will show that even if Res- $\mathbf{W}_{\mathbf{1}}$ and $\mathbf{R e s}-\mathbf{W}_{\mathbf{2}}$ are assumed, Extensionality is consistent with a violation of Ground in the case of unordered triplets.

The model is defined as follows:

Definition 3. The variable-domain $\mathrm{S} 5$ model $\mathfrak{M}_{2}$ is defined so that:

- $W$ contains two worlds $\left\{w, w^{\prime}\right\}$

- $D$ contains elements $\{\Lambda, a, s, t\}$ denoted by $\underline{\Lambda}, \underline{a}, \underline{s}, \underline{t}$, respectively

- $R=W \times W$

- $v$ provides an interpretation so that:

$-\mathcal{E}_{w}=D$ and $\mathcal{E}_{w^{\prime}}=\{\Lambda, a, t\}$

$-\varepsilon_{w}=\{\langle\Lambda, a\rangle,\langle\Lambda, s\rangle,\langle\Lambda, t\rangle,\langle a, s\rangle,\langle a, t\rangle,\langle s, t\rangle\}$

$-\varepsilon_{w^{\prime}}=\{\langle\Lambda, a\rangle,\langle\Lambda, t\rangle,\langle a, t\rangle\}$

A salient interpretation of the elements of $D$ at $w$ can be provided by interpreting $D$ as the first few von Neumann ordinals, i.e., a reading in which $\Lambda$ is understood as the empty set $\varnothing, a$ is interpreted as the singleton $\{\varnothing\}, s$ as the pair $\{\varnothing,\{\varnothing\}\}$, and $t$ as the triplet $\{\varnothing,\{\varnothing\},\{\varnothing,\{\varnothing\}\}\}$. Given the absence of $s$ in $\mathcal{E}_{w^{\prime}}$, however, $t$ plays the role of the pair $\{\varnothing,\{\varnothing\}\}$ at $w^{\prime}$.

First, let us confirm that we are working with an appropriate model. This requires that we check that the domains of both worlds are indeed models of Extensionality:

Observation 8. At both $w$ and $w^{\prime}$, Extensionality holds.

Proof. This may be easily confirmed by checking the extensions of the relation $\varepsilon$ at each world.

Now, we may confirm that the restricted assumptions Res- $\mathbf{W}_{\mathbf{1}}$ and $\mathbf{R e s}-\mathbf{W}_{\mathbf{2}}$ hold for $\mathfrak{M}_{2}$ by the following two observations: 
Observation 9. $w \Vdash \square(\underline{\Lambda}=\imath x . \neg \exists y(y \varepsilon x))$

Proof. By consulting the interpretation of $\varepsilon$, it is easy to confirm that at both $w$ and $w^{\prime}$ the only element to which no other element bears $\varepsilon$ is $\Lambda$. Hence, in every world, $\underline{\Lambda}=\imath x . \neg \exists y(y \varepsilon x)$ is true.

Observation 10. $w \Vdash \square(\underline{a}=\imath x . \forall y(y \varepsilon x \leftrightarrow(\mathcal{E}(y) \wedge(y=\underline{\Lambda})))$

Proof. As before, at both $w$ and $w^{\prime}, a$ is the unique element whose only "member" (i.e., element bearing relation $\varepsilon$ to $a$ ) is $\Lambda$. Hence, at both worlds, $\underline{a}=$ $\imath x . \forall y(y \varepsilon x \leftrightarrow(\mathcal{E}(y) \wedge(y=\underline{\Lambda}))$ is true, which in turn entails that the formula is necessarily true at $w$.

$\mathfrak{M}_{2}$ thus satisfies each of Wigglesworth's enthymematic assumptions, entailing that in this context, there is enough fodder for Wigglesworth's argument to go through. It follows that in $\mathfrak{M}_{2}$, Ground does in fact hold of singletons.

Observation 11. $w \Vdash \square[\neg \mathcal{E}(\underline{\Lambda}) \rightarrow \neg \exists y \forall x(x \varepsilon y \leftrightarrow(\mathcal{E}(x) \wedge x=\underline{\Lambda}))]$

Proof. At both $w$ and $w^{\prime}$, the sentence $\neg \mathcal{E}(\underline{\Lambda})$ is false, entailing that the conditional $\neg \mathcal{E}(\underline{\Lambda}) \rightarrow \neg \exists y \forall x(x \in y \leftrightarrow(\mathcal{E}(x) \wedge x=\underline{\Lambda}))$ is true at both worlds. As $w$ and $w^{\prime}$ exhaust the domain of worlds, this is a necessary truth, whence we infer that $\square(\neg \mathcal{E}(\underline{\Lambda}) \rightarrow \neg \exists y \forall x(x \varepsilon y \leftrightarrow(\mathcal{E}(x) \wedge x=\underline{\Lambda})))$ holds at both worlds.

As $a$ is the only singleton at either world, Ground holds for all singletons. In other words, granted the assumption that both Res- $\mathbf{W}_{\mathbf{1}}$ and $\mathbf{R e s}-\mathbf{W}_{\mathbf{2}}$ hold, we can assume that the singleton case of Ground would be correct.

Now, while the ontological dependence of singletons on their members is attractive, the thesis of Ground is more frequently encountered as the unqualified assertion that all sets ontologically depend on their members. While Wigglesworth refrains from offering a proof that this thesis holds in full generality, he suggests that the general structure of his argument should suffice to provide a demonstration of the unqualified thesis:

There is also reason to think that the argument presented here can be generalised to show that, beyond the case of singletons, all sets depend on their members, and that this dependence is asymmetric. 10. $174]$

However, $\mathfrak{M}_{2}$ witnesses that the assumptions $\mathbf{R e s}-\mathbf{W}_{\mathbf{1}}$ and $\mathbf{R e s}-\mathbf{W}_{\mathbf{2}}$ are insufficient to lift the validity of Ground to all sets. The lifting of the property to the set-theoretic multiverse in general will require stronger principles than Wigglesworth assumes. In particular, although $\mathfrak{M}_{2}$ satisfies both of the enthymematic assumptions employed by Wigglesworth, Ground fails in the case of unordered triplets.

As a principle about triplets, Ground states that if any one of three elements fails to exist, then the triplet containing them must fail to exist. A particular instance of Ground for triplets will thus be: 


$$
\square(\neg(\mathcal{E}(\underline{\Lambda}) \wedge \mathcal{E}(\underline{a}) \wedge \mathcal{E}(\underline{s})) \rightarrow \neg \exists y \forall x(x \varepsilon y \leftrightarrow(\mathcal{E}(x) \wedge(x=\underline{\Lambda} \vee x=\underline{a} \vee x=\underline{s})))
$$

As a result, if this sentence fails to hold in $\mathfrak{M}_{2}$, then Ground for triplets does not follow from Extensionality even when augmented by the additional premises Res- $\mathbf{W}_{\mathbf{1}}$ and Res- $\mathbf{W}_{\mathbf{2}}$.

Observation 12. At $w^{\prime}$, the sentence $\neg(\mathcal{E}(\underline{\Lambda}) \wedge \mathcal{E}(\underline{a}) \wedge \mathcal{E}(\underline{s}))$ is true.

Proof. Because $s \notin \mathcal{E}_{w^{\prime}}$, the formula $\mathcal{E}(\underline{s})$ fails, rendering the conjunction false and its negation true.

Observation 13. At $w^{\prime}, \neg(\exists y \forall x(x \varepsilon y \leftrightarrow(\mathcal{E}(x) \wedge(x=\underline{\Lambda} \vee x=\underline{a} \vee x=\underline{s}))))$ is false.

Proof. We begin by evaluating the sentence $\exists y \forall x(x \varepsilon y \leftrightarrow(\mathcal{E}(x) \wedge(x=\underline{\Lambda} \vee x=$ $\underline{a} \vee x=\underline{s}))$ ). Note first that the domain of quantification is finite and that each element of the domain is associated with a constant. Hence, when we instantiate the existential quantifier on $\underline{t}$, the truth of the universal formula $\forall x(x \in \underline{t} \leftrightarrow(\mathcal{E}(x) \wedge(x=\underline{\Lambda} \vee x=\underline{a} \vee x=\underline{s}))$ reduces to the truth of the conjunction of the following sentences:

- $\underline{\Lambda} \varepsilon \underline{t} \leftrightarrow(\mathcal{E}(\underline{\Lambda}) \wedge(\underline{\Lambda}=\underline{\Lambda} \vee \underline{\Lambda}=\underline{a} \vee \underline{\Lambda}=\underline{s}))$

- $\underline{a} \varepsilon \underline{t} \leftrightarrow(\mathcal{E}(\underline{a}) \wedge(\underline{a}=\underline{\Lambda} \vee \underline{a}=\underline{a} \vee \underline{a}=\underline{s}))$

- $\underline{t} \varepsilon \underline{t} \leftrightarrow(\mathcal{E}(\underline{t}) \wedge(\underline{t}=\underline{\Lambda} \vee \underline{t}=\underline{a} \vee \underline{t}=\underline{s}))$

In the first two cases, both $\underline{\Lambda} \varepsilon \underline{t}$ and $\underline{a} \varepsilon \underline{t}$ hold at $w^{\prime}$, entailing that the lefthand sides of the biconditionals are true. But both $\mathcal{E}(\underline{\Lambda})$ and $\mathcal{E}(\underline{a})$ hold - as well as the trivial identities $\underline{\Lambda}=\underline{\Lambda}$ and $\underline{a}=\underline{a}$ - and the right-hand sides of the respective biconditionals are also true. As both sides of the biconditionals have identical truth values, the biconditionals themselves are true at $w^{\prime}$.

In the third case, both $\underline{t} \varepsilon \underline{t}$ and the disjunction $\underline{t}=\underline{\Lambda} \vee \underline{t}=\underline{a} \vee \underline{t}=\underline{s}$ fail at $w^{\prime}$, whence both sides of the biconditional are false at $w^{\prime}$. Hence, $w^{\prime} \Vdash \underline{s} \varepsilon \underline{t} \leftrightarrow$ $(\mathcal{E}(\underline{s}) \wedge(\underline{s}=\underline{\Lambda} \vee \underline{s}=\underline{a} \vee \underline{s}=\underline{s}))$.

Thus, the formula $\exists y \forall x(x \in y \leftrightarrow(\mathcal{E}(x) \wedge(x=\underline{\Lambda} \vee x=\underline{a} \vee x=\underline{s})))$ is true - and its negation is false - at $w^{\prime}$.

These two observations immediately yield the failure of the aforementioned instance of Ground for triplets.

Observation 14. At w, the formula

$$
\square(\neg(\mathcal{E}(\underline{\Lambda}) \wedge \mathcal{E}(\underline{a}) \wedge \mathcal{E}(\underline{s})) \rightarrow \neg \exists y \forall x(x \varepsilon y \leftrightarrow(\mathcal{E}(x) \wedge(x=\underline{\Lambda} \vee x=\underline{a} \vee x=\underline{s})))
$$

is false.

Proof. By Observations 12 and 13 , we immediately infer that the sentence

$$
\neg(\mathcal{E}(\underline{\Lambda}) \wedge \mathcal{E}(\underline{a}) \wedge \mathcal{E}(\underline{s})) \rightarrow \neg \exists y \forall x(x \varepsilon y \leftrightarrow(\mathcal{E}(x) \wedge(x=\underline{\Lambda} \vee x=\underline{a} \vee x=\underline{s}))
$$


has a true antecedent and a false consequent and thus does not hold at $w^{\prime}$. Because $w R w^{\prime}, w^{\prime}$ serves as an accessible world at which the sentence fails, whence we conclude that

$$
\square(\neg(\mathcal{E}(\underline{\Lambda}) \wedge \mathcal{E}(\underline{a}) \wedge \mathcal{E}(\underline{s})) \rightarrow \neg \exists y \forall x(x \varepsilon y \leftrightarrow(\mathcal{E}(x) \wedge(x=\underline{\Lambda} \vee x=\underline{a} \vee x=\underline{s})))
$$

fails at $w$.

\subsection{Baptism by Definition and Rigid Designators}

We have shown that the task of establishing that Ground holds of sets in general - and not merely singletons - requires stronger principles than Wigglesworth assumes. In this section, we will consider strengthening the persistence criteria of Res- $\mathbf{W}_{\mathbf{1}}$ and Res- $\mathbf{W}_{\mathbf{2}}$ by extending them to a broader class of elements.

To return to the matter of the questionable inference, let us examine the circumstances under which it is introduced. Wigglesworth's defense of the stipulation that the definition $\imath x . \varphi(x)$ under which a proper name $\underline{t}$ is introduced should hold of the individual so named in all possible worlds appears in the below passages:

This stipulation [i.e., Res- $\mathbf{W}_{\mathbf{1}}$ ] is justified... because it ensures that we are talking about the singleton of $x$. If $\{x\}$ did not satisfy this condition, we would not be talking about the singleton of $x$; we would be talking about something else.[10, 167]

And:

$\left[\underline{s}^{\prime}\right]$ is defined as the set that has as its members all and only those objects that are identical to $[\underline{a}]$. As this is definitional of $\left[\underline{s}^{\prime}\right]$, it should hold in every world, including $w \cdot[10,173]$

Upon closer inspection, the underlying principle invoked in these passages seems to be a very unusual thesis. Wigglesworth appears to defend this inference on epistemic grounds by suggesting that when we fix the reference of a term by a description $7 x \cdot \varphi(x)$, then we would be unable to pick out its referent in another possible world without access to that description. This position suggests that - at least in certain cases - the description by which an individual term is introduced remains the only path to its referent.

This principle is obviously too strong to be applied to terms in general, as such a principle contradicts the very spirit of of rigid designation - a notion to which Wigglesworth himself appeals. It is part and parcel of rigid designation that once we have baptized a name by means of a description, the name itself suffices to refer to the individual in any possible world. The rigidity of rigid designation is precisely the thesis that after the initial baptism, no "guide" to the individual is required apart from the name itself.

Hence, if left unrestricted, the persistence criteria that would be employed in a suitable modification of the argument would lead to consequences that contradict some very deeply held theses about modality and descriptions. To use 
the familiar Kripkean example of [6], of the descriptions "the first Postmaster General of the United States" and "the inventor of bifocals," a simple argument by introducing proper names (e.g., "Postmaster General" and "Bifocal Inventor") would lead to the necessity of the statement:

- $\urcorner x . x$ is the first Postmaster General $=\imath y . y$ invented bifocals

Perhaps the apparent unsuitability of the unrestricted principle is due to the fact that Franklin is an object with which we are acquainted by ostension. The tenor of Wigglesworth's justification, on the other hand, seems to presuppose that sets must be introduced definitionally.

But extending the criteria to all objects introduced by definition is itself far too liberal, as there are objects whose denoting terms are fixed by definition and yet seem to resist the type of persistence criteria employed by Wigglesworth. A salient example, as Kripke notes in [6], is the case of Neptune. The reference of the term "Neptune" was fixed by means of a definitional description (rather than by ostension); we will continue to use the phrase "the planet causing such and such discrepancies in the orbits of certain other planets" as a stand-in for whatever Urbain Le Verrier employed. Now, suppose that one should encounter the following passage in a paper on metaphysics:

In any possible world, Neptune causes such and such discrepancies in the orbits of these planets because it ensures that we are talking about Neptune. If Neptune did not cause these discrepancies, we would not be talking about Neptune; we would be talking about something else.

Such a passage seems to contradict all but the most trivial notion of modality. If this passage is to be rejected as absurd, this suggests that more severe restrictions to such criteria are required.

\subsection{Purely Set-Theoretical Persistence Criteria}

Wigglesworth's justification for $\mathbf{R e s}-\mathbf{W}_{\mathbf{1}}$ and $\mathbf{R e s}-\mathbf{W}_{\mathbf{2}}$ appeals only to general intuitions about epistemology and intensionality, with no particularization to the case of mathematical objects. This seems to entail that the guiding principle to which Wigglesworth appeals is not restricted to merely mathematical entities.

Despite this, one might still object that the examples we have considered in this section differ from the cases of $\{a\}$ and $\varnothing$ insofar as they have made use of empirical predicates. One could then argue that more general persistence criteria fail in these cases due to, perhaps, the imprecision of empirical properties, although the application of such persistence criteria in the realm of mathematical objects holds without problems.

But such principles seem to fail in mathematical examples as well. Consider the following strengthened criterion: 
Res- $\mathbf{W}_{\mathbf{M}}$ For any term $\underline{t}$ introduced by a description $\underline{t}=\imath x \cdot \varphi(x)$ in the language of set theory, the formula $\varphi(\underline{t})$ is true in every possible world in which $\mathcal{E}(\underline{t})$ is true.

This seems to be the weakest generalization strong enough to ensure that Ground holds for all sets. In the particular case of set theory, very odd consequences remain. While such a criterion permits one to identify sufficiently many sets across possible worlds, the identification compelled by the adoption Res- $\mathbf{W}_{\mathbf{M}}$ leads to a nearly Platonistic conception of set.

The adoption of this principle, in effect, would be equivalent to the introduction of an axiom scheme such as:

Set Persistence $\exists x \forall y(y \varepsilon x \leftrightarrow \varphi(y)) \rightarrow\left(\underline{t}_{\varphi}=\imath x . \forall y(y \varepsilon x \leftrightarrow \varphi(y))\right)$

where $\underline{t}_{\varphi}$ is a constant schematically correlated with an open formula of set theory $\varphi(y)$. In plain language, the scheme entails that whenever $\varphi(y)$ defines a set $S$, there exists a term $\underline{t}_{\varphi}$ that is "definitional" of $S$ and guarantees the transworld identity of all witnesses to $\varphi(y)$.

This scheme appears at first blush to perform admirably. For example, the foregoing has produced two models in which instances of Ground fails - the model $\mathfrak{M}_{1}$ witnessed a failure of Ground for singletons while $\mathfrak{M}_{2}$ witnessed a failure of Ground for triplets. As counterexamples to elements of Wigglesworth's reasoning, any sufficient repairs to the argument must disqualify these two models, and the axiom eliminates these counterexamples insofar as there are instances of the axiom not satisfied by these models. When $\varphi(y)$ is defined as follows:

- $\varphi_{1}(y)=_{d f} \mathcal{E}(y) \wedge y=\underline{a}$

$\mathfrak{M}_{1}$ clearly does not verify this instance of Set Persistence. The validity of this instance would entail that both $w \Vdash \underline{t}_{\varphi_{1}}=\underline{a}$ and $w^{\prime} \Vdash \underline{t}_{\varphi_{1}}=\underline{s}$ hold. From this, one would be able to infer that $w \Vdash \underline{a}=\underline{s}$, contradicting the the distinctness of $a$ and $s$.

Set Persistence is similarly effective against $\mathfrak{M}_{2}$ with the selection of $\varphi(y)$ in which:

- $\varphi_{2}(y)=_{d f} \mathcal{E}(y) \wedge(y=\underline{\Lambda} \vee y=\underline{a})$

Recall that in $\mathfrak{M}_{2}, s$ serves as the unordered pair comprising elements $\Lambda$ and $a$ at $w$ and that this role is played by $t$ at $w^{\prime}$. The validity of this instance of Res- $\mathrm{W}_{\mathrm{M}}$ would thus require that both $w \Vdash \underline{t}_{\varphi_{2}}=\underline{s}$ and $w^{\prime} \Vdash \underline{t}_{\varphi_{2}}=\underline{t}$, from which one could infer easily that $w \Vdash \underline{s}=\underline{t}$. Hence, the adoption of a persistence principle like Res- $\mathbf{W}_{\mathbf{M}}$ rules out $\mathfrak{M}_{2}$ as a countermodel as well.

But it can easily be seen that this axiom has some unfortunate consequences. Consider, for example, Gödel's constructible universe $\mathbf{L}$, iteratively constructed by taking the definable subsets of earlier stages. Accepting Set Persistence entails that the constructible universe $\mathbf{L}$ and the von Neumann universe $\mathbf{V}$ cannot both be models of ZF, with the force of metaphysical necessity. 
The set $\mathbf{V}_{\omega}$ is definable, so there is a definite description in the language of set theory that corresponds to the formula $x=\wp\left(\mathbf{V}_{\omega}\right) !^{3}$ Let " $x \sqsubseteq y$ " serve as an abbreviation for the formula $\forall z(z \varepsilon x \rightarrow z \varepsilon y)$. Then the following is an instance of Set Persistence:

$$
\text { - } \exists x \forall y\left(y \varepsilon x \leftrightarrow y \sqsubseteq \mathbf{V}_{\omega}\right) \rightarrow\left(\underline{t}=\imath x . \forall y\left(y \varepsilon x \leftrightarrow y \sqsubseteq \mathbf{V}_{\omega}\right)\right.
$$

That is, if there exists a power set of $\mathbf{V}_{\omega}$, then $\underline{t}$ denotes that set. Both $\mathbf{L}$ and $\mathbf{V}$ make true the antecedent of this sentence, so should Set Persistence be assumed, both make true $\underline{t}=\imath x$. $\left(y \varepsilon x \leftrightarrow y \sqsubseteq \mathbf{V}_{\omega}\right)$, i.e., $\underline{t}=\mathbf{V}_{\omega+1}$.

In any discussion of possible worlds and models of set theory, it seems that (presuming that ZF has a model), $\mathbf{L}$ and $\mathbf{V}$ ought to be considered paradigm cases of possible ways that sets could be. Now, suppose that one constructs an S5 model in which both $\mathbf{L}$ and $\mathbf{V}$ are possible worlds corresponding to their statuses as "possible models" of ZF. Then as $\underline{t}$ denotes $\wp\left(\mathbf{V}_{\omega}\right)$ in both $\mathbf{L}$ and in $\mathbf{V}$, the same object must serve as the power set of $\mathbf{V}_{\omega}$, that is, $\underline{t}$ rigidly designates the same set in all possible worlds (in which "world" is taken to be "possible model of ZF").

But this is false; what $\mathbf{L}$ thinks answers to $\wp\left(\mathbf{V}_{\omega}\right)$-i.e., the set $\mathbf{L}_{\omega+1}$-is not identical to what $\mathbf{V}$ thinks is $\wp\left(\mathbf{V}_{\omega}\right)$. If one assumes the validity of Set Persistence, then, there is no variable-domain S5 model in which both $\mathbf{L}$ and $\mathbf{V}$ are included in the domain of possible worlds 4

Now, recall that Wigglesworth is drawing metaphysical conclusions from this formalism. If we follow Wigglesworth and take seriously the metaphysical upshot of the foregoing observations, then accepting a principle like Res- $\mathbf{W}_{\mathbf{M}}$ entails that it is metaphysically impossible that both $\mathbf{L}$ or $\mathbf{V}$ are models of set theory. If one wishes to say with a straight face that $\mathbf{L}$ and $\mathbf{V}$ - or other models, e.g., HOD - are coherent ways that the set-theoretical universe could be, one must reject Set Persistence. In other words, if Res- $\mathbf{W}_{\mathbf{M}}$ does not outright entail Platonism, it certainly runs dangerously close to Platonism. The persistence criterion severely restricts the class of possible models of set theory. For example, if one were to live in a pointwise definable universe - a model of set theory in which every set was definable without parameters (cf. [3]) - then Res$\mathbf{W}_{\mathbf{M}}$ would quite clearly entail the metaphysical impossibility of the existence of an alternative model. In other words, a kind of set-theoretical actualism would follow.

Although we stop well short of proof, the foregoing considerations raise the specter of a dilemma facing attempts to repair Wigglesworth's argument. If the appropriate persistence criteria are too weak and do not hold for every set, then the foregoing results suggest there will remain sets that do not ontologically depend on their members. On the other hand, if one is to guarantee that all

\footnotetext{
${ }^{3}$ As the least inductive set, $\omega$ is definable in the language of set theory and for any definable ordinal $\alpha, \mathbf{V}_{\alpha}$ and $\mathbf{L}_{\alpha}$ are similarly definable. As the power set function is also definable, there exists a formula in the language of set theory amounting to $x=\wp\left(\mathbf{V}_{\omega}\right)$. Cf. [5].

${ }^{4}$ Properly speaking, $\mathbf{L}$ and $\mathbf{V}$ are proper classes and S5 models are regarded as sets. However, this example can be rephrased as a prohibition against the sets $\mathbf{L}_{\omega+2}$ and $\mathbf{V}_{\omega+2}$ appearing as possible worlds in a variable-domain S5 model.
} 
sets enjoy this ontological dependence, then the persistence criteria must be strengthened to hold of the entire set-theoretic universe. In this case, one will be forced to cordon off important (and prima facie coherent) corners of the set-theoretic multiverse as metaphysically impossible.

\section{Conclusions}

The example of a set's being ontologically dependent on its members is entrenched for a reason. Despite the fact that metaphysical grounding can at times seem esoteric, the set-theoretical case provides a very salient and compelling illustration of grounding. It is convenient that the theory of sets is well-studied, as the clarity that the formalism of set theory provides has the potential to aid in the clarification of grounding as a whole. Hence, it is likely that, properly executed, the program initiated by Wigglesworth in [10 may ultimately bear more heavily on the general notion of grounding and the program is thus worthy of further development.

Moreover, independently of the metaphysical notion of grounding, the framework of modal logic has been fruitful in set theory proper. Kripke semantics and possible worlds have been employed to examine large cardinal properties in Andreas Blass' 1, to explore the notion of forcing in Hamkins and Benedikt Löwe's [4, and to help elucidate the construction of Cohen models in Raymond Smullyan and Melvin Fitting's 9]. There is a tradition going back a quarter of a century in which models of ZF and possible worlds have been harmoniously deployed for purely mathematical pursuits. This suggests that deploying the two in tandem for metaphysical pursuits has the potential to bear fruit as well.

In other words, the foregoing is not intended as an objection to the program that Wigglesworth has initiated but rather as an observation that its implementation is in need of revision. The mathematical evidence suggests that there exists an adequate modal framework within which we may pose not only questions about the grounding of sets, but of grounding in general. However, the observations in this paper suggest that there remain a number of unsolved and subtle problems before an adequate framework is reached.

\section{References}

[1] Blass, A. Infinitary combinatorics and modal logic. Journal of Symbolic Logic 55, 2 (1990), 761-778. http://www.jstor.org/stable/2274663.

[2] Fine, K. Ontological dependence. Proceedings of the Aristotelian Society 95 (1995), 269-290. http://www.jstor.org/stable/4545221.

[3] Hamkins, J., Linetsky, D., And Reitz, J. Pointwise definable models of set theory. Journal of Symbolic Logic 78, 1 (2013), 139-156. http: //projecteuclid.org/euclid.jsl/1358951104. 
[4] Hamkins, J., AND Löwe, B. The modal logic of forcing. Transactions of the American Mathematical Society 360, 4 (2008), 1793-1817. http://www.ams.org/journals/tran/2008-360-04/ S0002-9947-07-04297-3/S0002-9947-07-04297-3.pdf

[5] JEch, T. Set Theory, Third Millenium ed. Springer, New York, 2003.

[6] KrIPKe, S. Naming and Necessity. Harvard University Press, Cambridge, MA, 1980.

[7] Priest, G. An Introduction to Non-Classical Logic: From If to Is, second ed. Cambridge University Press, New York, 2008.

[8] Schaffer, J. Monism: The priority of the whole. Philosophical Review 119, 1 (2010), 31-76. http://philreview.dukejournals.org/content/ $119 / 1 / 31$.

[9] Smullyan, R., And Fitting, M. Set Theory and the Continuum Problem, vol. 34 of Oxford Logic Guides. Oxford University Press, Oxford, 1996.

[10] Wigglesworth, J. Set-theoretic dependence. Australasian Journal of Logic 12, 3 (2015), 150-176. http://ojs.victoria.ac.nz/ajl/article/ view/2131/3276. 